\title{
Metal-free 2D/2D black phosphorus and covalent triazine framework heterostructure for $\mathrm{CO}_{2}$ photoreduction
}

\author{
Jian Li ${ }^{a, b, 1}$, Peng Liu ${ }^{a, 1}$, Hongliang Huanga,*, Yang Li ${ }^{a}$, Yuanzhe Tang, ${ }^{a, b}$ Donghai Mei ${ }^{a, *}$, \\ Chongli Zhong ${ }^{\mathrm{a}, \mathrm{b}, \mathrm{c}, *}$
}

a State Key Laboratory of Membrane Separation and Membrane Processes, School of Chemistry and Chemical Engineering, Tiangong University, Tianjin 300387, P. R. China

${ }^{b}$ State Key Laboratory of Organic-Inorganic Composites, Beijing University of Chemical Technology,

Beijing 100029, P. R. China

${ }^{c}$ Beijing Advanced Innovation Center for Soft Matter Science and Engineering, Beijing University of Chemical Technology, Beijing 100029, P. R. China

${ }^{1}$ These authors contributed equally.

*Corresponding authors: H. L. Huang, huanghongliang@tiangong.edu.cn;

D. Mei,dhmei@tiangong.edu.cn;

C. L. Zhong, zhongchongli@tiangong.edu.cn

Number of pages: 17

Number of Figures: 14

Number of Tables: 2 


\section{Contents}

1. The characterization of $\mathrm{CTF}$

2. The characterization of CTF-BP

3. The photocatalytic reduction of $\mathrm{CO}_{2}$

4. The optical property of CTF and CTF-BP

5. The CTF-BP heterojunction structure used in the DFT calculations

6. Comparison of the photocatalytic $\mathrm{CO}_{2}$ reduction performance over the CTF-BP heterostructure composite with literature reported results

7. Reference 
1. The characterization of CTF.

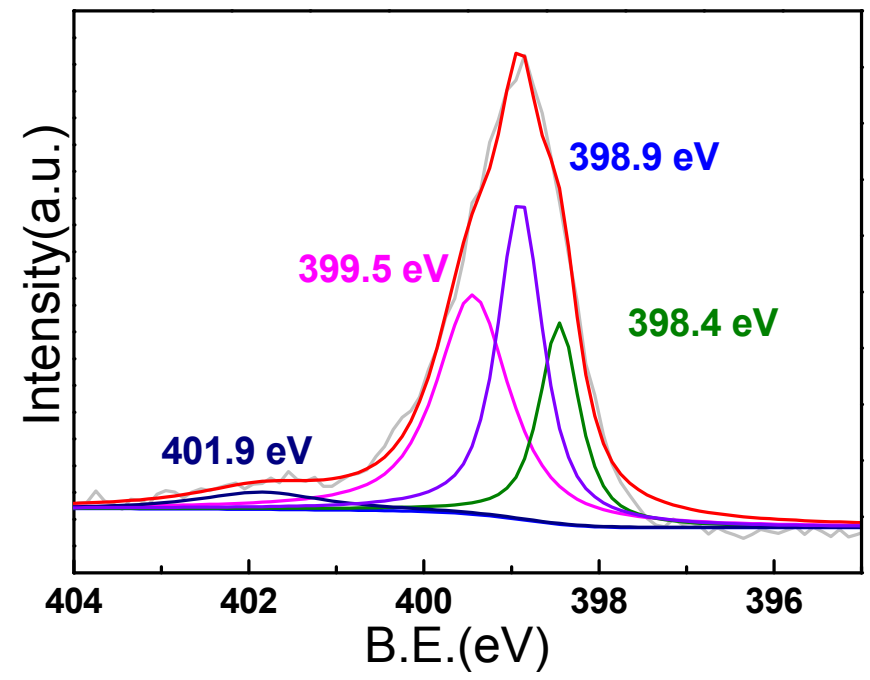

Figure S1. N 1s XPS spectra of CTF.

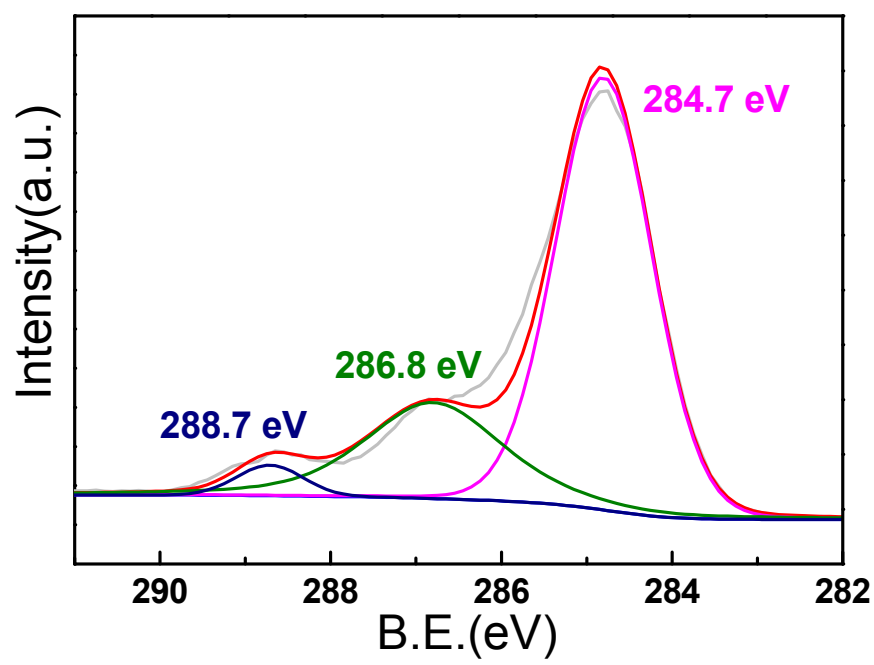

Figure S2. C 1s XPS spectra of CTF. 


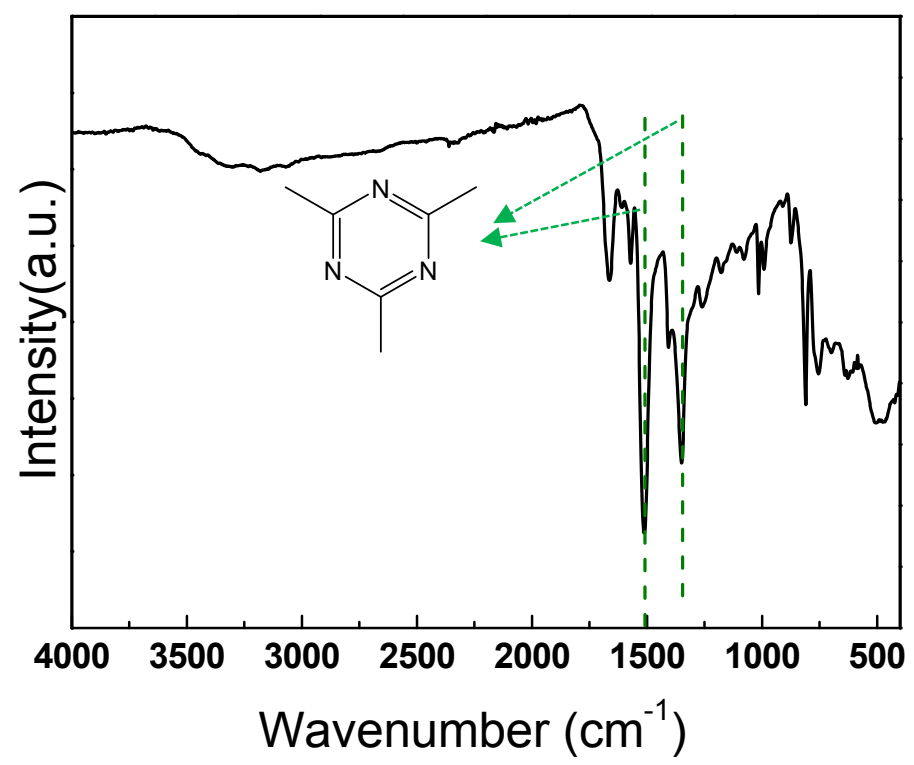

Figure S3. FT-IR spectra of CTF.

2. The characterization of CTF-BP.

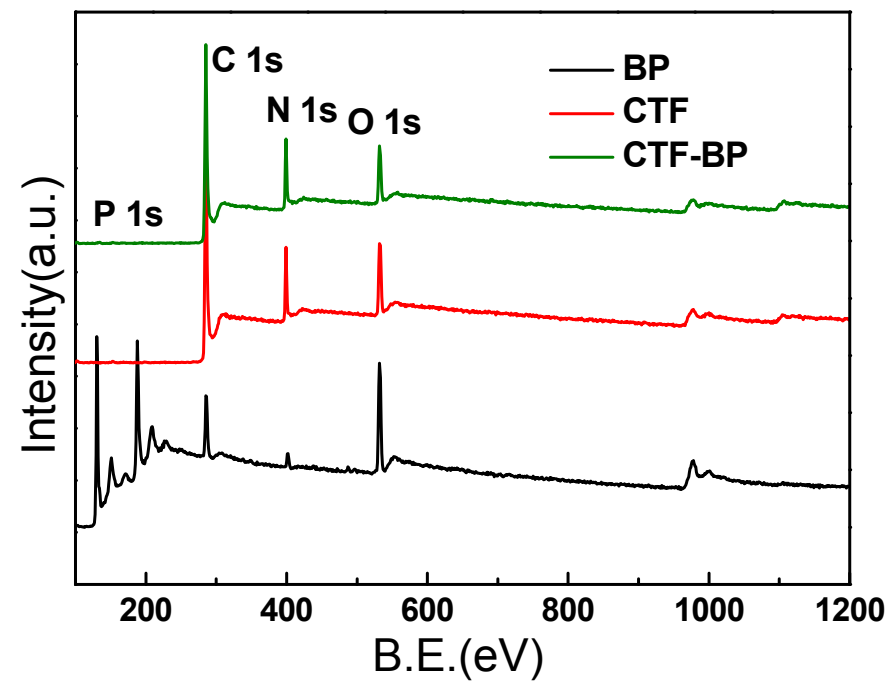

Figure S4. XPS full spectra of BP, CTF, and CTF-BP. 


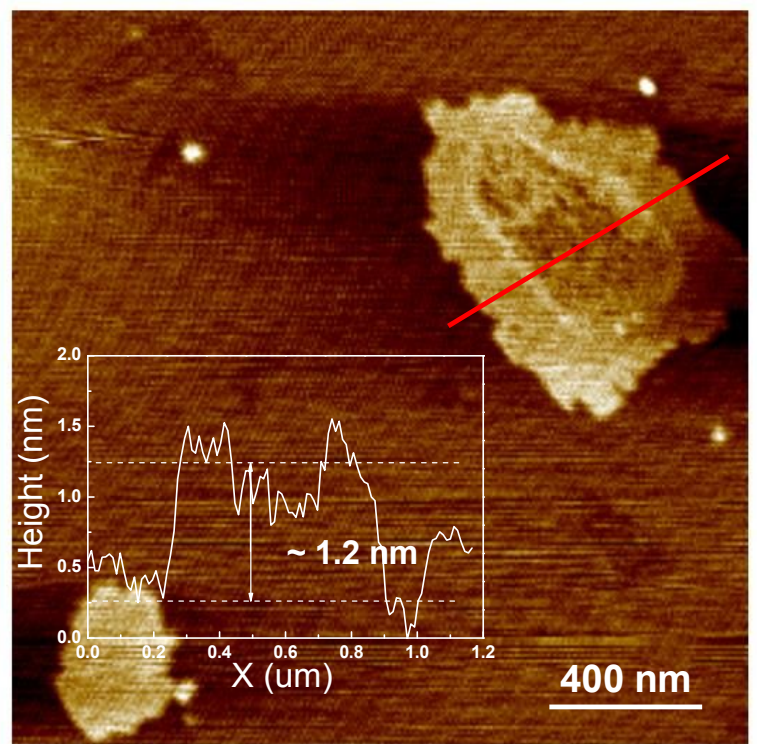

Figure S5. AFM image of CTF-BP.

3. The photocatalytic reduction of $\mathrm{CO}_{2}$.

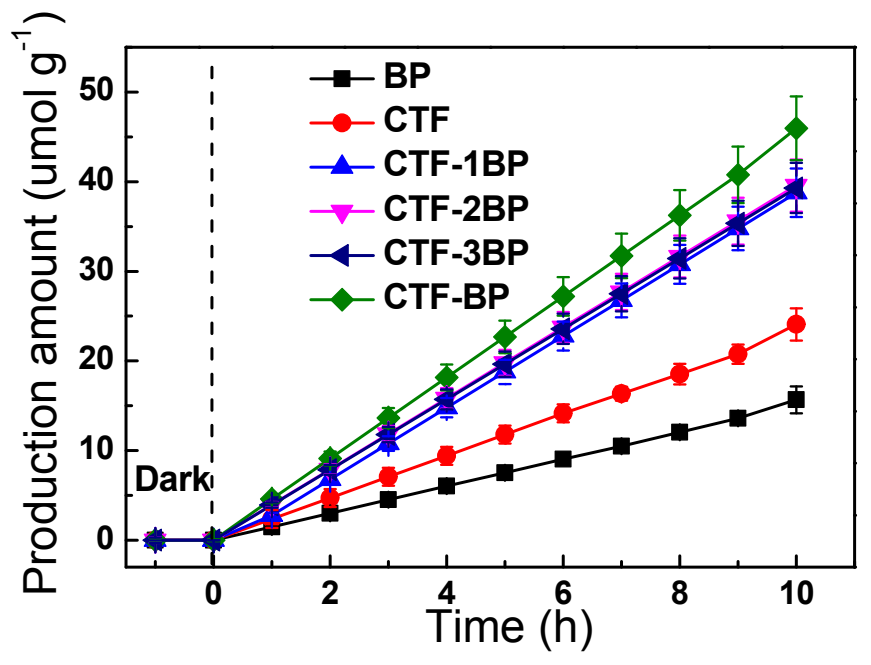

Figure S6. Time-dependent CO production rates over BP, CTF, and CTF-BP catalysts in the photocatalytic $\mathrm{CO}_{2}$ reduction. 


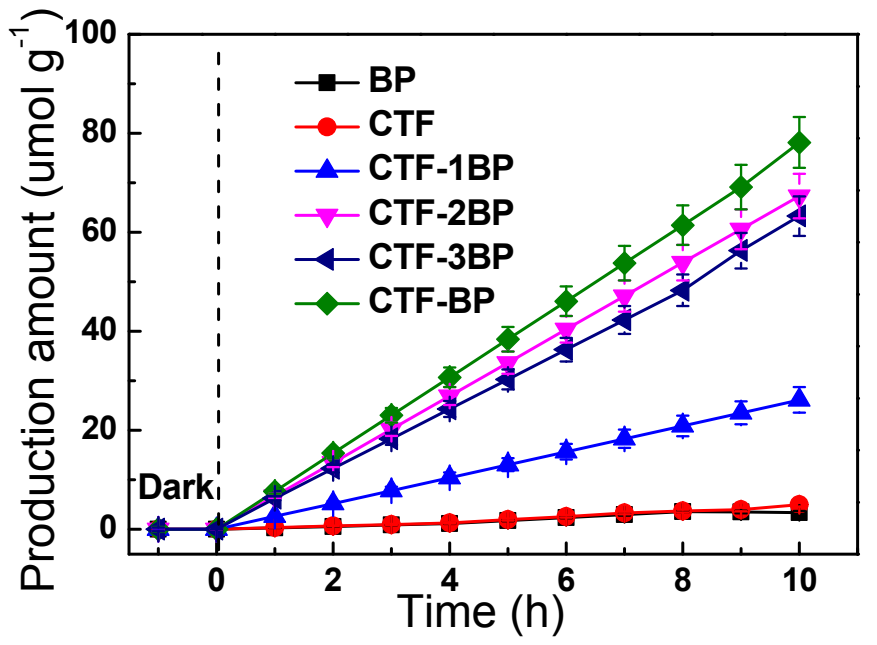

Figure S7. Time-dependent $\mathrm{CH}_{4}$ production rates over BP, CTF, and CTF-BP catalysts in the photocatalytic $\mathrm{CO}_{2}$ reduction.

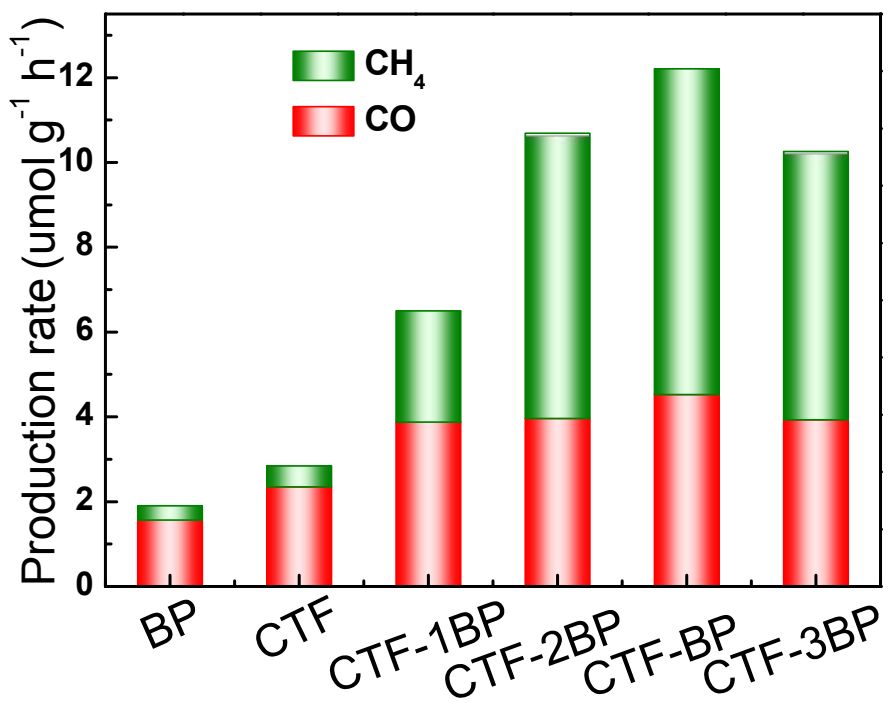

Figure S8. Average production rates of $\mathrm{CO}$ and $\mathrm{CH}_{4}$ in the photocatalytic $\mathrm{CO}_{2}$ reduction. 


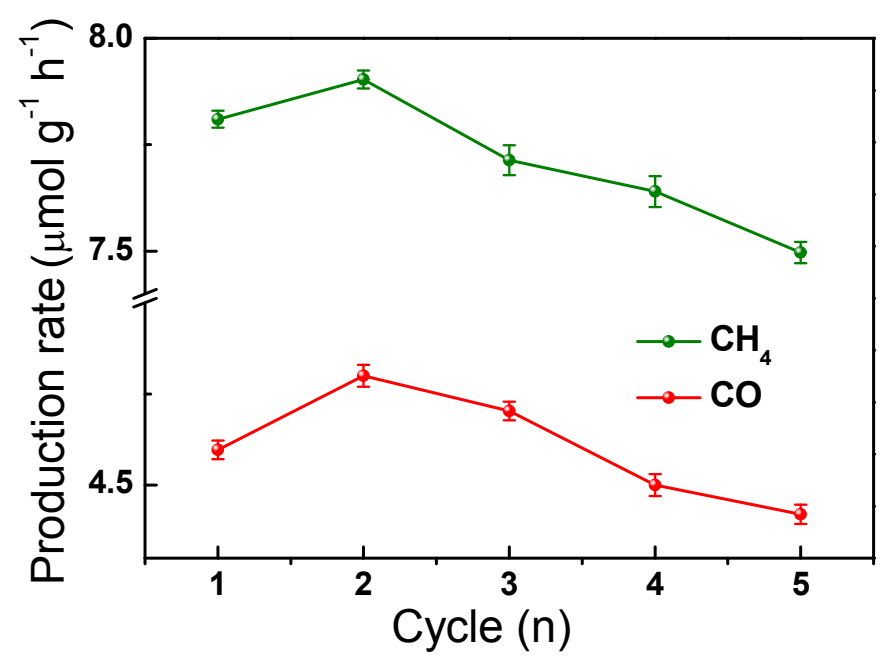

Figure S9. The recycle experiments of CTF-BP catalyst in the photocatalytic $\mathrm{CO}_{2}$ reduction.

\section{The optical property of CTF and CTF-BP.}

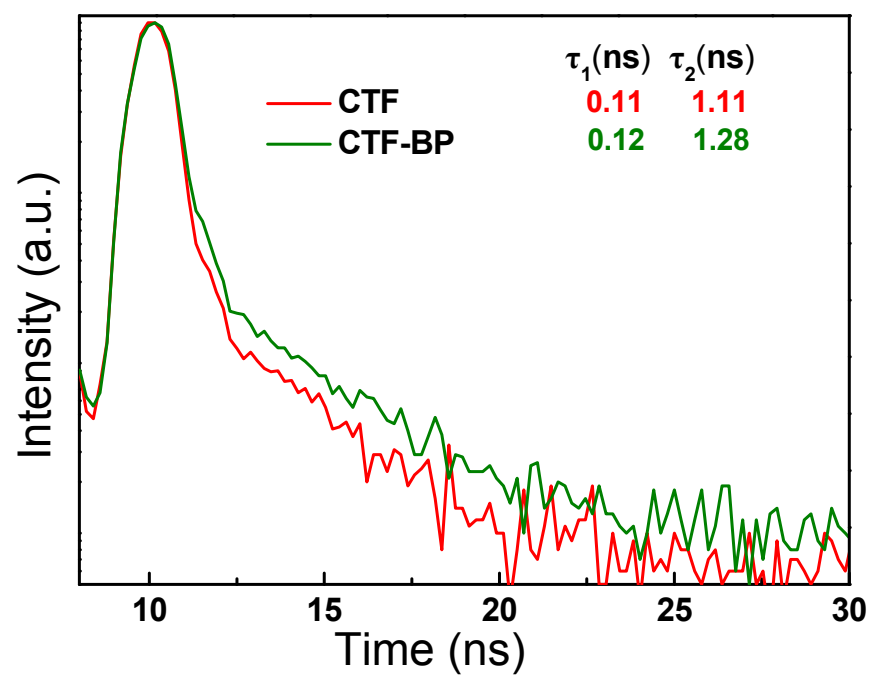

Figure S10. Time-resolved fluorescence decay spectra of CTF and CTF-BP at $390 \mathrm{~nm}$ excitation. 


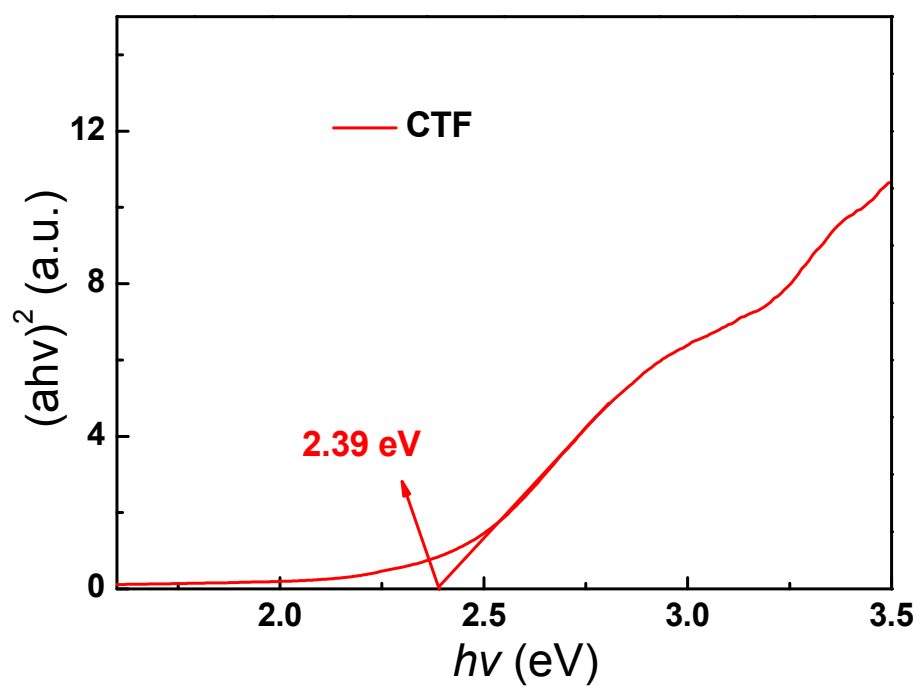

Figure S11. The Tauc plot of CTF.

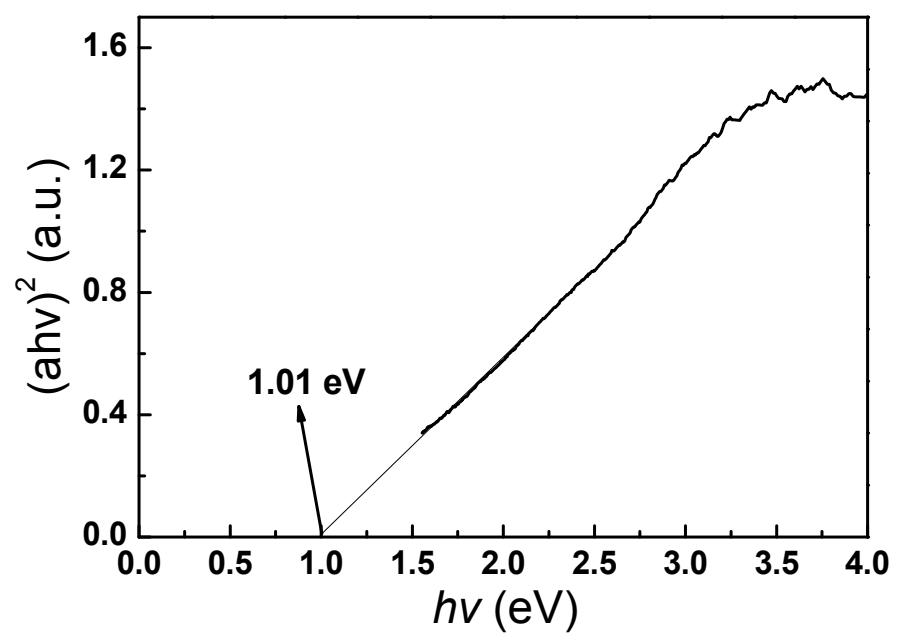

Figure S12. The Tauc plot of BP. 


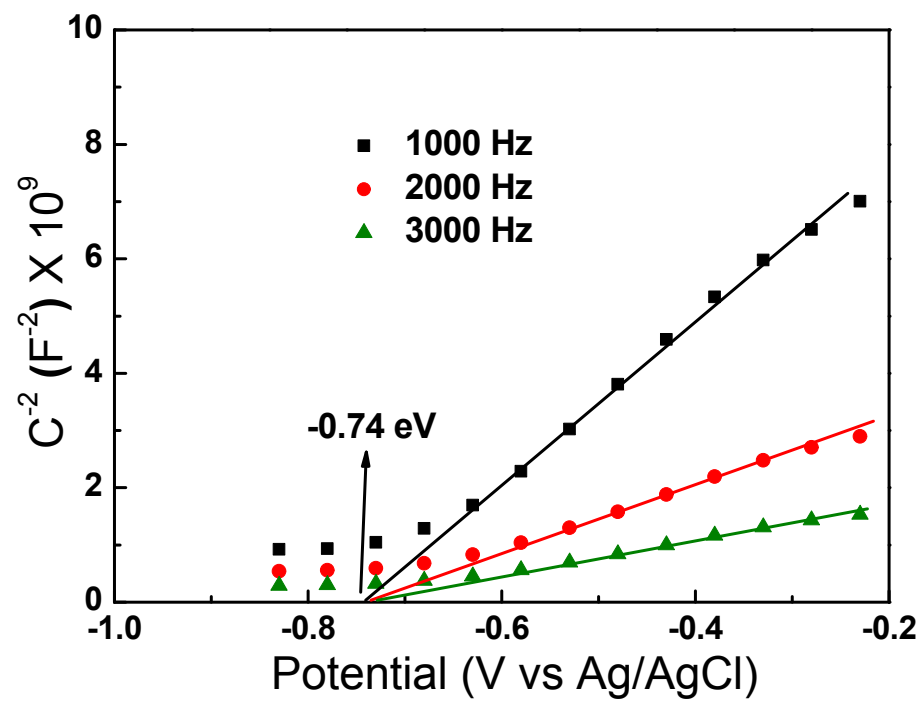

Figure S13. Mott-Schottky plots of CTF.

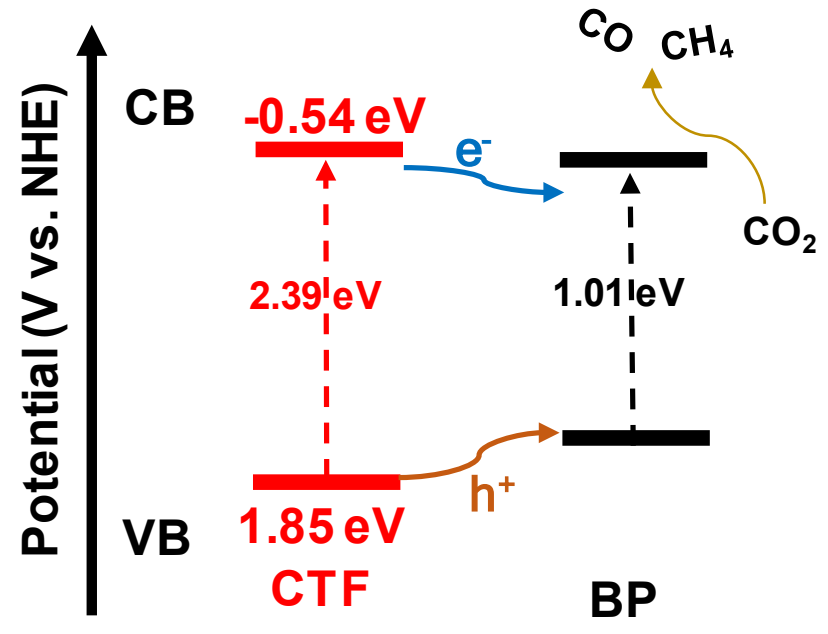

Type I heterojunction

Figure S14. Schematic energy level diagrams of CTF and BP.

\section{The CTF-BP heterojunction structure used in the DFT calculations.}

Table S1. The atomic Cartesian positions of the optimized CTF-BP heterojunction.

CTF-BP

1.00000000000000

22.1867008209000005

0.0000000000000000

0.0000000000000000 


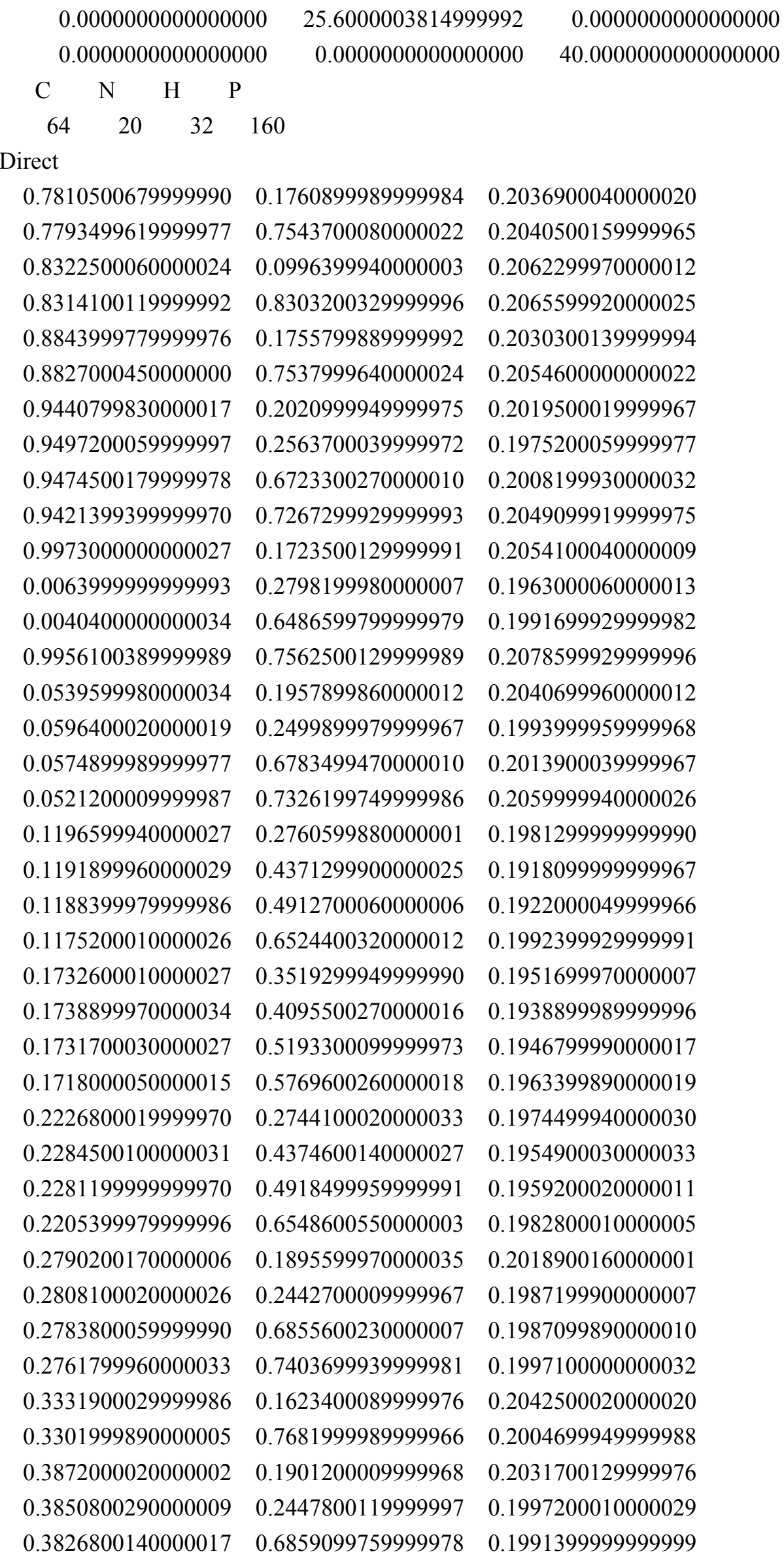




\begin{tabular}{|c|c|c|}
\hline 0.3844000209999976 & 0.7408700029999977 & 0.2001399990000010 \\
\hline 0.4430499990000030 & 0.2753399830000021 & 0.1987900020000026 \\
\hline 4360300150000000 & 0.4382299939999967 & 0.1958500029999968 \\
\hline 4356199899999993 & 0.4926399810000035 & 0.1956499930000035 \\
\hline 0.4409700000000001 & 0.6556799909999995 & 0.1990000010000017 \\
\hline 0.4921900129999983 & 0.3530999970000011 & 0.1970000029999994 \\
\hline 0.4910199870000014 & 0.4107800060000031 & 0.1960899950000012 \\
\hline 0.4902499700000007 & 0.5205799929999984 & 0.1957599999999999 \\
\hline 0.4907399889999979 & 0.5782499820000027 & 0.1967300060000028 \\
\hline 0.5461500429999973 & 0.2774499869999971 & 3399979999992 \\
\hline 0.5455100109999975 & 0.4388799829999996 & 0.1958899970000019 \\
\hline 0.5451100009999976 & 0.4930100139999993 & 0.1957100030000021 \\
\hline 0.5440499709999997 & 0.6542599850000030 & 0.1990399960000033 \\
\hline 0.6116300150000029 & 0.1972199949999975 & 0.2054899930000005 \\
\hline 0.606180 & 0.2514100040000002 & 0.2007 \\
\hline 0.6040400199999993 & 0.6802800199999979 & 0.2002500059999974 \\
\hline 0.6097299910000018 & 0.7345100380000034 & 0.2048700089999969 \\
\hline 0.6682400119999983 & 0.1736200019999998 & 0.2065000059999988 \\
\hline 0.6595700000000022 & 0.2810400119999983 & 0.1974699969999989 \\
\hline 0.6571400249999968 & 0.6503300270000025 & 0.1973399999999970 \\
\hline 0.6663900209999980 & 0.7577999570000031 & 0.2060800080000007 \\
\hline 0.7215799969999992 & 0.2030800069999970 & 0.2029299969999983 \\
\hline 0.7161800399999976 & 0.2574199960000030 & 0.1985899929999988 \\
\hline 0.7139600000000002 & 0.6736200200000013 & 0.1987799999999993 \\
\hline 0.7196399979999981 & 0.7278900490000026 & 0.2029299969999983 \\
\hline 0.1190899939999994 & 0.3284599959999994 & 0.1964100000000002 \\
\hline 0.1174099939999991 & 0.5999600430000029 & 0.1973099949999977 \\
\hline 0.1705600009999984 & 0.24704999899999979 & 0.1988899950000018 \\
\hline 0.1680400070000019 & 0.6818199800000002 & 0.1994199989999998 \\
\hline 0.4953599999999980 & 0.2481899929999969 & 0.1998600009999976 \\
\hline 0.4930700250000015 & 0.6831800089999973 & 0.2001699919999993 \\
\hline 0.5465400369999998 & 0.3298300080000018 & 0.1976299999999966 \\
\hline 0.5447699970000031 & 0.6019199919999991 & 0.1967499969999977 \\
\hline 0.7787600489999988 & 0.1233800019999975 & 0.2056799889999965 \\
\hline 0.7776500290000001 & 0.8071499939999995 & 0.2047100070000027 \\
\hline 0.8329200419999978 & 0.2039400090000001 & 0.2023499969999989 \\
\hline 0.8308699600000011 & 0.7259499919999968 & 0.2042799950000003 \\
\hline 0.8860300199999998 & 0.1228000019999982 & 0.2048300029999979 \\
\hline 0.8848899970000019 & 0.8066200339999980 & 0.2069399829999981 \\
\hline 0.2264399889999993 & 0.3266199999999984 & 0.1955800060000001 \\
\hline 0.2248299999999972 & 0.6027200360000009 & 0.1971199990000017 \\
\hline 0.3328499989999969 & 0.2712999800000020 & 0.1976599930000020 \\
\hline 0.3307600070000021 & 0.6588600480000011 & 0.1984899999999996 \\
\hline 0.4391300249999972 & 0.3275699860000003 & 0.1975200059999977 \\
\hline
\end{tabular}




$\begin{array}{llll}0.4374199909999987 & 0.6034399850000014 & 0.1977999929999967 \\ 0.8320000109999981 & 0.0569399999999973 & 0.2079900029999990 \\ 0.8316500350000027 & 0.8730699740000034 & 0.2078599929999996 \\ 0.9090099820000006 & 0.2799400090000006 & 0.1949200030000000 \\ 0.9065899799999997 & 0.6488500439999996 & 0.1986299989999978 \\ 0.9933099619999979 & 0.1303600029999998 & 0.2094399929999966 \\ 0.0103300000000033 & 0.3218899989999997 & 0.1927600029999965 \\ 0.0076299999999989 & 0.6065199809999982 & 0.1957899929999982 \\ 0.9919200300000028 & 0.7983000460000014 & 0.2115299940000028 \\ 0.0772000030000015 & 0.4151999890000013 & 0.1901900049999981 \\ 0.0765399989999977 & 0.5128199899999970 & 0.1908199909999979 \\ 0.0946099949999990 & 0.1723199870000016 & 0.2071599960000015 \\ 0.0929299960000023 & 0.7560899739999982 & 0.2082999940000008 \\ 0.2354900019999988 & 0.1698300030000013 & 0.2027799840000029 \\ 0.2325199980000008 & 0.7598700260000015 & 0.2001399990000010 \\ 0.2706499900000026 & 0.4156299989999965 & 0.1968999979999992 \\ 0.2700400040000019 & 0.5140099970000023 & 0.1974799870000012 \\ 0.3333800139999994 & 0.1199499969999991 & 0.2072799919999966 \\ 0.3299400000000006 & 0.8108200010000033 & 0.2016799929999991 \\ 0.3940499839999987 & 0.4160200000000032 & 0.1959599970000028 \\ 0.3933399739999999 & 0.5144299860000032 & 0.1957899929999982 \\ 0.4307400109999975 & 0.1706299909999984 & 0.2049400089999978 \\ 0.4277999879999967 & 0.7607799660000012 & 0.2008399959999991 \\ 0.5709300059999975 & 0.1737899869999993 & 0.2086100100000010 \\ 0.5691300049999981 & 0.7580000049999995 & 0.2080100060000021 \\ 0.5878800360000014 & 0.4173600239999971 & 0.1960999969999975 \\ 0.5871999849999980 & 0.5149400159999971 & 0.1958699939999988 \\ 0.3163219990\end{array}$




\begin{tabular}{|c|c|c|}
\hline 0.4174799890000003 & 0.0621300000000033 & 0.2858600140000007 \\
\hline 0.6853799889999976 & 0.9999099970000032 & 0.2895699979999975 \\
\hline 7158700390000021 & 0.0002799999999965 & 0.3433599950000001 \\
\hline 7831100350000000 & 0.0631199999999978 & 0.3438000200000033 \\
\hline 0.6179100279999972 & 0.0620400009999997 & 0.2884099959999986 \\
\hline 0.8839899959999968 & 0.9996499720000003 & 0.2910299779999974 \\
\hline 0.9175399949999985 & 0.0001400000000018 & 0.3442299840000018 \\
\hline 0.9846199619999965 & 0.0631000050000026 & 0.3431000229999981 \\
\hline 0.8171399940000015 & 0.0621399970000027 & 0.2907199859999992 \\
\hline 0.0820199990000035 & 0.1244800050000023 & 0.2873199940000006 \\
\hline 0.1181200050000015 & 0.1258500089999970 & 0.3399300100000033 \\
\hline 0.1851899990000021 & 0.1884799979999983 & 0.3377599949999990 \\
\hline 0.0148299989999998 & 0.1868800049999990 & 0.2882600069999981 \\
\hline 0.2836999920000025 & 0.1244100070000016 & 0.2850500110000027 \\
\hline 0.3165900130000026 & 0.1256899979999986 & 0.3384200100000001 \\
\hline 0.3837000259999996 & 0.1882900080000027 & 0.3385599850000034 \\
\hline 0.2163499990000020 & 0.1868600000000029 & 0.2840399979999972 \\
\hline 0.4848200100000000 & 0.1245400009999997 & 0.2859899999999982 \\
\hline 0.5154600310000035 & 0.1252000019999997 & 0.3398999930000031 \\
\hline 0.5825099950000023 & 0.1881500120000013 & 0.3410099979999970 \\
\hline 0.4175200079999968 & 0.1868600000000029 & 0.2854900119999968 \\
\hline 0.6848600109999978 & 0.1245599969999986 & 0.2889199729999987 \\
\hline 0.7159799920000012 & 0.1259600070000033 & 0.3424799920000012 \\
\hline 0.7829300180000018 & 0.1888999869999992 & 0.3417200089999994 \\
\hline 0.6166899680000029 & 0.1862999960000025 & 0.2880599980000014 \\
\hline 0.8843799480000030 & 0.1243999949999974 & 0.2901999950000018 \\
\hline 0.9174700169999994 & 0.1259900049999985 & 0.3434500219999990 \\
\hline 0.9846699960000009 & 0.1889300129999967 & 0.3419399739999989 \\
\hline 0.8165199920000035 & 0.1862200139999999 & 0.2886199949999977 \\
\hline 0.0827699949999996 & 0.2487300109999993 & 0.2852100129999968 \\
\hline 0.1179899999999989 & 0.2511099869999995 & 0.3379800080000024 \\
\hline 0.1850899969999986 & 0.3135899780000031 & 0.3356699939999999 \\
\hline 0.0157099999999986 & 0.3115099990000019 & 0.2842899799999969 \\
\hline 0.2844299899999996 & 0.2488400000000013 & 0.2834300040000031 \\
\hline 0.3163200090000018 & 0.2508400159999979 & 0.3368900059999973 \\
\hline 0.3833200039999980 & 0.3134800070000026 & 0.3352600099999989 \\
\hline 0.2163499990000020 & 0.3109399929999981 & 0.2821500060000020 \\
\hline 0.4853399879999998 & 0.2488200140000032 & 0.2846499919999985 \\
\hline 0.5155400250000000 & 0.2510400079999968 & 0.3384700060000014 \\
\hline 0.5827999660000032 & 0.3135400219999980 & 0.3373000140000002 \\
\hline 0.4172400089999968 & 0.3108499900000012 & 0.2822099919999985 \\
\hline 0.6840000290000035 & 0.2486799989999966 & 0.2860899929999974 \\
\hline 0.7156800059999995 & 0.2514100040000002 & 0.3395699980000018 \\
\hline 0.7833400000000026 & 0.3134300139999979 & 0.3379800080000024 \\
\hline
\end{tabular}




$\begin{array}{llll}0.6163999979999986 & 0.3109399929999981 & 0.2841300010000012 \\ 0.8841599550000012 & 0.2483200060000001 & 0.2863900179999987 \\ 0.9170799799999969 & 0.2513299840000016 & 0.3398300169999970 \\ 0.9844800059999983 & 0.3136200040000006 & 0.3379299879999991 \\ 0.8167600149999998 & 0.3108300040000032 & 0.2847399950000025 \\ 0.0831900029999986 & 0.3740699909999989 & 0.2818300249999979 \\ 0.1176600000000008 & 0.3757300170000022 & 0.3349600080000030 \\ 0.1851799840000012 & 0.4377600110000017 & 0.3341499810000030 \\ 0.0164700010000018 & 0.4373599899999974 & 0.2819199799999978 \\ 0.2829500060000001 & 0.3741599940000029 & 0.2798099990000011 \\ 0.3160599980000001 & 0.3756299929999969 & 0.3331600189999975 \\ 0.3831300140000025 & 0.4378400310000004 & 0.3329899789999970 \\ 0.2151600070000015 & 0.4368599809999978 & 0.2804000140000014 \\ 0.4839699999999993 & 0.3740199969999978 & 0.2814100029999977 \\ 0.5152999590000036 & 0.3756499980000001 & 0.3350599999999986 \\ 0.5827999660000032 & 0.4375999729999975 & 0.3350100039999973 \\ 0.4171399860000022 & 0.4372399980000026 & 0.2799400090000006 \\ 0.6834099869999974 & 0.3739000059999995 & 0.2828600169999973 \\ 0.7157499839999986 & 0.3753600209999988 & 0.3363299849999990 \\ 0.7831599829999973 & 0.4374600140000027 & 0.3364200119999978 \\ 0.6166399780000020 & 0.4371100230000025 & 0.2818900110000016 \\ 0.8834600030000033 & 0.3740100130000030 & 0.2835999970000032 \\ 0.9172099640000013 & 0.3759000020000016 & 0.3368000030000005 \\ 0.9850300299999972 & 0.4376799919999996 & 0.3355999950000026 \\ 0.8165700260000008 & 0.4371200060000007 & 0.2831900119999986 \\ 0.0834299990000034 & 0.5005900109999999 & 0.2811500070000008 \\ 0.1176200040000026 & 0.4998399990000024 & 0.3343000169999968 \\ 0.1851799840000012 & 0.5618500249999983 & 0.3343699930000028 \\ 0.7815600\end{array}$




$\begin{array}{llll}0.8165799980000017 & 0.5632799779999971 & 0.2842600110000006 \\ 0.0826200019999987 & 0.6265800350000035 & 0.2837199929999983 \\ 0.1178399960000007 & 0.6240699720000009 & 0.3365700009999983 \\ 0.1854200069999976 & 0.6862299789999966 & 0.3370000119999972 \\ 0.0148299989999998 & 0.6886500020000028 & 0.2862499949999986 \\ 0.2834300089999999 & 0.6258299850000029 & 0.2807700159999982 \\ 0.3165200139999982 & 0.6237900160000009 & 0.3341400150000027 \\ 0.3838799999999978 & 0.6860300049999992 & 0.3361799959999985 \\ 0.2161899910000002 & 0.6886099919999964 & 0.2833000179999985 \\ 0.4844299729999975 & 0.6256999730000032 & 0.2824399949999972 \\ 0.5152800139999982 & 0.6237499699999987 & 0.3361899849999972 \\ 0.5823500090000024 & 0.6863300400000014 & 0.3388299940000010 \\ 0.4172900000000013 & 0.6883599510000025 & 0.2829999920000006 \\ 0.6851500240000021 & 0.6258999459999970 & 0.2851200100000000 \\ 0.7159900070000020 & 0.6233699900000005 & 0.3387799739999977 \\ 0.7833899479999999 & 0.6858000060000009 & 0.3410399910000024 \\ 0.6182900069999988 & 0.6886400180000010 & 0.2862800119999989 \\ 0.8832299520000007 & 0.6264399639999993 & 0.2853400230000034 \\ 0.9171399860000022 & 0.6237700120000014 & 0.3384900089999974 \\ 0.9846699960000009 & 0.6860600309999967 & 0.3399699929999969 \\ 0.8158699869999992 & 0.6888999689999977 & 0.2875800130000030 \\ 0.0827000009999992 & 0.7506700490000000 & 0.2869800089999970 \\ 0.1182899960000015 & 0.7488399630000018 & 0.3396599769999966 \\ 0.1851500029999968 & 0.8117800079999995 & 0.3388600110000013 \\ 0.0149100009999970 & 0.8126699800000026 & 0.2887699839999982 \\ 0.2841500130000014 & 0.7504900429999992 & 0.2848199840000021 \\ 0.3169899800000024 & 0.7488800470000001 & 0.3380199910000030 \\ 0.3841599980000012 & 0.8115299669999985 & 0.3379600049999993 \\ 0.2163899959999966 & 0.8126000190000013 & 0.2851600170000026 \\ 0.8 .817850\end{array}$




$\begin{array}{lll}0.0150799999999975 & 0.9377100179999971 & 0.2895900010000005 \\ 0.2832899879999999 & 0.8752699810000024 & 0.2853300089999991 \\ 0.3170200039999997 & 0.8741500100000010 & 0.3384900089999974 \\ 0.3835200090000015 & 0.9372799699999987 & 0.3391399859999993 \\ 0.21571000899999866 & 0.9374599759999995 & 0.2860300059999972 \\ 0.4845299959999991 & 0.8752699810000024 & 0.2858400109999977 \\ 0.5154299849999973 & 0.8743300160000018 & 0.3396199940000031 \\ 0.5824600050000015 & 0.9370399869999986 & 0.3413599969999979 \\ 0.4172299940000030 & 0.9377100179999971 & 0.2858999970000013 \\ 0.6857000050000011 & 0.8749899880000029 & 0.2895900010000005 \\ 0.7160099950000003 & 0.8744999640000017 & 0.3434500219999990 \\ 0.7830600019999991 & 0.9374400089999995 & 0.3440299990000000 \\ 0.6184600090000032 & 0.9373099960000033 & 0.2887499810000023 \\ 0.8838600130000032 & 0.8751299850000009 & 0.2908099890000031 \\ 0.9176999820000020 & 0.8746299760000014 & 0.3439399960000031 \\ 0.9850199720000035 & 0.9374200410000029 & 0.3435600039999969 \\ 0.8167499569999990 & 0.9374700349999969 & 0.2908799889999969\end{array}$

\section{Comparison of the photocatalytic $\mathrm{CO}_{2}$ reduction performance over the CTF-BP}

\section{heterostructure composite with literature reported results}

Table S2. Comparison of the photocatalytic $\mathrm{CO}_{2}$ reaction performance for the CTF-BP catalyst with other recently reported metal-free catalysts.

\begin{tabular}{|c|c|c|c|c|c|}
\hline Catalysts & $\begin{array}{c}\text { light } \\
\text { source }\end{array}$ & $\begin{array}{c}\mathrm{CO} \\
\text { production } \\
\text { rate }\left(\mathrm{umol} \cdot \mathrm{g}^{-}\right. \\
\left.{ }^{1} \cdot \mathrm{h}^{-1}\right)\end{array}$ & $\begin{array}{c}\mathrm{CH}_{4} \\
\text { production } \\
\text { rate }\left(\mathrm{umol} \cdot \mathrm{g}^{-}\right. \\
\left.{ }^{1} \cdot \mathrm{h}^{-1}\right)\end{array}$ & $\begin{array}{c}\text { Total } \\
\text { electron } \\
\text { transfer } \\
\left(\mathrm{umol} \cdot \mathrm{g}^{-}\right. \\
\left.1 \cdot \mathrm{h}^{-1}\right)\end{array}$ & Reference \\
\hline $\mathrm{CTF-BP}$ & $\lambda>420 \mathrm{~nm}$ & 4.60 & 7.81 & 71.68 & $\begin{array}{c}\text { This } \\
\text { work }\end{array}$ \\
\hline $\mathrm{CCN}$ & $\begin{array}{c}\text { Full } \\
\text { spectrum }\end{array}$ & 1.25 & 6.35 & 53.3 & {$[1]$} \\
\hline $\begin{array}{c}\mathrm{BPQDs} / \mathrm{g}- \\
\mathrm{C}_{3} \mathrm{~N}_{4}\end{array}$ & $\begin{array}{c}\text { Full } \\
\text { spectrum }\end{array}$ & 6.54 & 0.29 & 15.4 & {$[2]$} \\
\hline $\mathrm{g}_{-} \mathrm{C}_{3} \mathrm{~N}_{4}$ & $\begin{array}{c}\text { Full } \\
\text { spectrum }\end{array}$ & 2.65 & 0.67 & 10.66 & \\
\hline
\end{tabular}




\begin{tabular}{|c|c|c|c|c|c|}
\hline PEosinY-1 & $\lambda>420 \mathrm{~nm}$ & 33 & $/$ & 66 & {$[3]$} \\
\hline $\mathrm{N}_{3}-\mathrm{COF}$ & $800 \mathrm{~nm} \geq$ & $/$ & 0.57 & 4.56 & {$[4]$} \\
& $\begin{array}{c}\lambda \geq 420 \\
\mathrm{~nm}\end{array}$ & & & & \\
& & & & \\
\hline
\end{tabular}

* Total electron transfer $=2 \times \mathrm{CO}$ production rate $+8 \times \mathrm{CH}_{4}$ production rate

\section{References}

[1] P. Xia, M. Antonietti, B. Zhu, T. Heil, J. Yu, S. Cao, Designing defective crystalline carbon nitride to enable selective $\mathrm{CO}_{2}$ photoreduction in the gas phase, Adv. Funct. Mater. 2019, 29, 1900093-1900102, DOI 10.1002/adfm.201900093.

[2] C. Han, J. Li, Z. Ma, H. Xie, G.I.N. Waterhouse, L. Ye, T. Zhang, Black phosphorus quantum dot/g- $\mathrm{C}_{3} \mathrm{~N}_{4}$ composites for enhanced $\mathrm{CO}_{2}$ photoreduction to $\mathrm{CO}, \mathrm{Sci}$. China Mater. 2018, 61, 1159-1166, DOI 10.1007/s40843-018-9245-y.

[3] X. Yu, Z. Yang, B. Qiu, S. Guo, P. Yang, B. Yu, H. Zhang, Y. Zhao, X. Yang, B. Han, Z. Liu, Eosin Y-functionalized conjugated organic polymers for Visiblelight-driven $\mathrm{CO}_{2}$ reduction with $\mathrm{H}_{2} \mathrm{O}$ to $\mathrm{CO}$ with high efficiency, Angew. Chem. Int. Ed. 2019, 58, 632-636, DOI 10.1002/anie.201812790.

[4] Y. Fu, X. Zhu, L. Huang, X. Zhang, F. Zhang, W. Zhu, Azine-based covalent organic frameworks as metal-free visible light photocatalysts for $\mathrm{CO}_{2}$ reduction with $\mathrm{H}_{2} \mathrm{O}$, Appl. Catal. $B$ Environ. 2018, 239, 46-51, DOI 10.1016/j.apcatb.2018.08.004. 